J. Appl. Math. \& Informatics Vol. 32(2014), No. 1 - 2, pp. 1 - 6 http://dx.doi.org/10.14317/jami.2014.001

\title{
A NEW VERTEX-COLORING EDGE-WEIGHTING OF COMPLETE GRAPHS
}

\author{
MOHAMMAD REZA FARAHANI
}

\begin{abstract}
Let $G=(V ; E)$ be a simple undirected graph without loops and multiple edges, the vertex and edge sets of it are represented by $V=$ $V(G)$ and $E=E(G)$, respectively. A weighting $w$ of the edges of a graph $G$ induces a coloring of the vertices of $G$ where the color of vertex $v$, denoted $S_{v}:=\sum_{e \ni v} w(e)$. A k-edge-weighting of a graph $G$ is an assignment of an integer weight, $w(e) \in\{1,2, \ldots, k\}$ to each edge $e$, such that two vertexcolor $S_{v}, S_{u}$ be distinct for every edge $u v$. In this paper we determine an exact 3-edge-weighting of complete graphs $k_{3 q+1} \forall q \in \mathbb{N}$. Several open questions are also included.
\end{abstract}

AMS Mathematics Subject Classification : 05C15, 05C78.

Key words and phrases: Graph labeling, Edge-weighting, Vertex-coloring, Complete Graph.

\section{Introduction}

Let $G=(V ; E)$ be a simple undirected graph without loops and multiple edges, the vertex and edge sets of it are represented by $V=V(G)$ and $E=E(G)$, respectively. A weighting $w$ of the edges of a graph $G$ induces a coloring of the vertices of $G$.

A $k$-edge-weighting of a graph $G$ is an assignment of an integer weight, $w(e) \in$ $\{1,2, \ldots, k\}$ to each edge $e$. The edge-weighting is proper if for every edge $e=u v$ incident a proper vertex-coloring and the colors of two vertices $u, v$ are distinct, where the color of a vertex $v$ is defined as the sum of the weights on the edges incident to that vertex. Clearly a graph cannot have a k-edge-weighting and vertex-coloring if it has a component which is isomorphic to $K_{2}$ i.e., an edge component. Throughout this paper, we denoted the color of a vertex $v$ by $S_{v}:=\sum_{u \in V(G)} w(u v)$, such that if $v w$ is not in $V(G) w(v w)=0$.

In particular a 3-edge-weighting of $G$ called 1-2-3-edge weighting and vertex coloring of $G$.

Received March 17, 2013. Revised May 30, 2013. Accepted July 3, 2013.

(c) 2014 Korean SIGCAM and KSCAM. 
In 2002 [9], Karonski, Euczak and Thomason conjectured that every graph without an edge component permits a 1-2-3-edge weighting and vertex coloring and proved their conjecture for the case of 3-colorable graphs [9]. For $k=2$ is not sufficient as seen for instance in complete graphs and cycles of length not divisible by 4 .

In 2004, they proved a graph without an edge component permits an 213edge-weighting and vertex coloring. In continue a constant bound of $k=30$ was proved by Addario-Berry, et.al in 2007 [1]. In next year, Addario-Berry's group improved this bound to $k=16$ [2] and also in 2008, T. Wang and $Q$. $Y u$ improved $k$ to 13 [10]. Recently, its new bounds are $k=5$ and $k=6$ by Kalkowski, et.al [7, 8]. In addition, for further study and more historical details, readers can see the recent papers [3-5].

In this paper, we show that there is a proper 1-2-3-edge weighting and vertex coloring for the complete graphs $K_{3 q+1} \forall q \in \mathbb{N}$ and obtain an exact weighting $w$ of all edges $e \in E\left(K_{3 q+1}\right)$ and alternatively a proper color of all vertices $v \in V\left(K_{3 q+1}\right)$. We present these main results in the following theorem:

Theorem 1.1. For the complete graph $K_{3 q+1}$ for every integer number $q$ with the vertex set $V\left(K_{3 q+1}\right)=\left\{v_{1}, v_{2}, \ldots, v_{3 q+1}\right\}$, there are a 3-edge weighting $w$ : $E\left(K_{3 q+1}\right) \rightarrow\{1,2,3\}$ and a vertex-coloring $s: V\left(K_{3 q+1}\right) \rightarrow\{9 q, 9 q-1, \ldots, 7 q+$ $1,7 q, 7 q-2,7 q-5,7 q-9, \ldots, 3 q+11,3 q+7,3 q+4\}$.

\section{Main Results and Algorithm}

For a graph complete graph $K_{n}=\left(V\left(K_{n}\right) ; E\left(K_{n}\right)\right)$, a 3-edge weighting is a function $w: E\left(K_{n}\right) \rightarrow\{1,2,3\}$ such that $S_{v}=\sum_{u \in V\left(K_{n}\right)} w(u v) \neq S_{u}$ is a color for any every vertex $v \in V\left(K_{n}\right)$. The edge weighting $w$ implies that $E\left(K_{n}\right)=E\left(K_{n}\right)_{1} \cup E\left(K_{n}\right)_{2} \cup E\left(K_{n}\right)_{3}$. Throughout this paper, we denoted the size of edge sets $E\left(K_{n}\right)_{1}, E\left(K_{n}\right)_{2}$ and $E\left(K_{n}\right)_{3}$ by $\gamma_{n}, \beta_{n}$ and $\alpha_{n}$, respectively. Obviously $\gamma_{n}+\beta_{n}+\alpha_{n}=\frac{n(n-1)}{2}$.

Before proving Theorem 1.1, for a general representation of complete graph $K_{3 q+1} \forall q \in \mathbb{N}$, we present a proper 3-edge weighting for all edges incident to a vertex $v$ in $3 q+1$ following steps and obtain all summations $S_{v}$.

2.1.Algorithm for 1-2-3-edge weighting and vertex coloring of $K_{3 q+1}$ $(q \geq 5)$ : At first, we denote all vertices of $K_{3 q+1}$ by $v_{1}, v_{2}, \ldots, v_{3 q+1}$, respectively. Obviously $E\left(K_{3 q+1}\right)=\left\{v_{i} v_{j} \mid i \neq j, i, j=1,2, \ldots, 3 q+1\right\}$ and this implies that $S_{v_{i}}=\sum_{v_{j} \in V\left(K_{3 q+1}\right), i \neq j, j=1, \ldots, 3 q+1} w\left(v_{i} v_{j}\right)=\left(S_{i}\right)$. Suppose $\forall i=$ $1,2, \ldots, 3 q+1 ; w\left(v_{i} v_{i}\right)=0$. So, we have

$\operatorname{Step}(1)$ - For the vertex $v_{1}$ label all its edges with $3\left(\forall v_{j} \in V\left(K_{3 q+1}\right) w\left(v_{1} v_{j}\right)=\right.$ 3 and $\left.S_{v_{1}}=\sum_{u \in V\left(K_{3 q+1}\right)} w(u v)=3(3 q)\right)$.

Step $(2)$ - For $v_{2}$ label all $v_{2}$ 's edges with 3 , except an edge $v_{2} v_{3 q+1}$, then $\forall j=1,3,4, \ldots, 3 q, w\left(v_{2} v_{j}\right)=3$ and $w\left(v_{2} v_{3 q+1}\right)=2$. Thus $S_{2}=S_{1}-1=9 q-1$.

Step(3)- For $v_{3}$ label all edges $v_{3} v_{j}(j=1,2,4, \ldots, 3 q-1)$ with 3 and $v_{3} v_{3 q}, v_{3} v_{3 q+1}$ with 2 . Thus $S_{3}=S_{2}-1=9 q-2$. 
Step(4)- For $v_{4}$ label all edges $v_{4} v_{j}(j=1,2,3,5, \ldots, 3 q-1)$ with $3, v_{4} v_{3 q}$ with 2 and $v_{4} v_{3 q+1}$ with 1 . Thus $S_{4}=S_{3}-1=9 q-3$.

Step(s)- $\forall s=5,7, \ldots, 2 q-1$ label all edges $v_{s} v_{j}(j=1,2, \ldots, s-1, s+$ $\left.1, \ldots, 3 q-\left[\frac{s}{2}\right]\right)$ with 3 , label $v_{s} v_{3 q-\left[\frac{s}{2}\right]+1}, v_{s} v_{3 q-\left[\frac{s}{2}\right]+2}$ with 2 and all edge $v_{s} v_{j}$ $\left(j=3 q-\left[\frac{s}{2}\right]+3, \ldots, 3 q+1\right)$ with 1 . Thus $S_{s}=3 \times\left(3 q-\left[\frac{s}{2}\right]-1\right)+2 \times 2+1 \times$ $\left(\left[\frac{s}{2}\right]-1\right)=9 q-2\left[\frac{s}{2}\right]=S_{s-2}-2$.

Step(r)- $\forall r=6,8, \ldots, 2 q$ label all edges $v_{r} v_{j}(j=1,2, \ldots, r-1, r+1, \ldots, 3 q+$ $\left.1-\left[\frac{r}{2}\right]\right)$ with 3 , label $v_{r} v_{3 q-\left[\frac{r}{2}\right]+2}$ with 2 and all edge $v_{r} v_{j}\left(j=3 q-\left[\frac{r}{2}\right]+3, \ldots, 3 q+\right.$ 1) with 1 . Thus $S_{r}=3 \times\left(3 q+1-\left[\frac{r}{2}\right]-1\right)+2 \times 1+1 \times\left(\left[\frac{r}{2}\right]-1\right)=9 q+1-r=S_{r-2}-2$.

$\operatorname{Step}(2 \mathrm{q}+1)$ - For $v_{2 q+1}$, all edges $v_{2 q+1} v_{j}(j=1,2, \ldots, 2 q)$ were labeled with 3. Thus label all edge $v_{2 q+1} v_{j}(j=2 q+2, \ldots, 3 q+1)$ with 1 . Thus $S_{2 q+1}=$ $3 \times 2 q+2 \times 0+1 \times(q)=7 q=S_{2 q}-1$.

$\operatorname{Step}(2 q+2)$ - For $v_{2 q+2}$, all edges $v_{2 q+2} v_{j}(j=1,2, \ldots, 2 q-2)$ were labeled with 3 , the edge $v_{2 q+2} v_{2 q-1}, v_{2 q+2} v_{2 q}$ were labeled with 2 and $v_{2 q+2} v_{2 q+1}$ were labeled with 1 . Thus label all edges $v_{2 q+2} v_{j}(j=2 q+3, \ldots, 3 q+1)$ with 1 and $S_{2 q+2}=3 \times(2 q-2)+2 \times 2+1 \times(q)=7 q-2=S_{2 q+1}-3$.

$\operatorname{Step}(\mathrm{t})-\forall t=2 q+3, \ldots, 3 q-2$ all edges $v_{t} v_{j}(j=1, \ldots, 6 q+2-2 t)$ were labeled with 3 , three edges $v_{t} v_{6 q+2-2 t+i}$ for $i=1,2,3$ were labeled with 2 and all edges $v_{t} v_{j}(j=6 q-2 t+6, \ldots, t-1)$ were labeled with 1 . Thus label all edges $v_{t} v_{j}$ $(j=t+1, \ldots, 3 q+1)$ with 1 and $S_{t}=3 \times(6 q+2-2 t)+2 \times 3+1 \times(2 t-3 q-5)=$ $15 q+7-4 t=S_{t-1}-4$.

$\operatorname{Step}(3 \mathrm{q}-1)-v_{3 q-1}, v_{3 q-1} v_{j}(j=1,2,3,4)$ were labeled with 3 and $v_{3 q-1} v_{5}$, $v_{3 q-1} v_{6}, v_{3 q-1} v_{7}$ were labeled with 2 and all edge $v_{3 q-1} v_{j}(j=8, \ldots, 3 q-2)$ were labeled with 1 . Thus label $v_{3 q-1} v_{3 q}, v_{3 q-1} v_{3 q+1}$ with 1 and $S_{3 q-1}=3 \times 4+$ $2 \times 3+1 \times(3 q-7)=3 q+11=S_{3 q-2}-4$.

$\operatorname{Step}(3 \mathrm{q})$ - For $v_{3 q}, v_{3 q} v_{1}, v_{3 q} v_{2}$ were labeled with 3 and $v_{3 q} v_{3}, v_{3 q} v_{4}, v_{3 q} v_{5}$ were labeled with 2 and all edge $v_{3 q} v_{j}(j=6, \ldots, 3 q-1)$ were labeled with 1 . Thus label the edge $v_{3 q+1} v_{3 q}$ with 1 and $S_{3 q}=3 \times 2+2 \times 3+1 \times(3 q-5)=3 q+7=$ $S_{3 q-1}-4$.

Step $(3 q+1)$ - Obviously, for the vertex $v_{3 q+1}$, the edge $v_{3 q+1} v_{1}$ were labeled with 3 and $v_{3 q+1} v_{2}, v_{3 q+1} v_{3}$ were labeled with 2 and all edge $v_{3 q+1} v_{j}(j=$ $4, \ldots, 3 q)$ were labeled with 1 . Thus $S_{3 q+1}=3+2 \times 2+1 \times(3 q-3)=3 q+4=$ $S_{3 q}-3$.

Now, we start the proof of main theorem as follow.

Proof. Let $K_{3 q+1}$ be a complete graph as order $3 q+1$ for every integer number $q$, with the vertex set $V\left(K_{3 q+1}\right)=\left\{v_{1}, v_{2}, \ldots, v_{3 q+1}\right\}$ and the edge set $E\left(K_{3 q+1}\right)=$ $\left\{e_{i j}=v_{i} v_{j} \mid v_{i}, v_{j} \in V\left(K_{3 q+1}\right)\right\}\left(\left|V\left(K_{3 q+1}\right)\right|=3 q+1\right.$ and $\left.\left|E\left(K_{3 q+1}\right)\right|=\frac{3 q(3 q+1)}{2}\right)$. It is easy to see that the above edge-weighting is a nice and proper 3-edge weighting $w$ (or 1-2-3-edge weighting and vertex coloring) of $K_{3 q+1}(q \geq 1)$. Because $\forall v_{i} \in V\left(K_{3 q+1}\right)$ and for every edge $e_{i j}=v_{i} v_{j}$ incident to $v_{i}$, we have an integer weight $w\left(v_{i} v_{j}\right) \in\{1,2,3\}$ such that this weighting naturally induces two distinct vertex coloring $S_{v_{i}}$ and $S_{v_{j}}$ to vertices $v_{i}, v_{j}$. 
An every edge $e_{i j}=v_{i} v_{j}(i, j=1,2, \ldots, 3 q+1, i \geq j)$ weighted in $\operatorname{Step}(i)$ of above 3-edge weighting $w$. Also, from above 3 -edge weighting $w$, one can see that all vertex color belong to the color set $\{9 q, 9 q-1, \ldots, 7 q+1,7 q, 7 q-2,7 q-$ $5,7 q-9, \ldots, 3 q+11,3 q+7,3 q+4\}$ (For example, see Figure 1, 2 and 3 ). In Figure 1, 2 and 3, a proper 1-2-3-edge weighting and vertex coloring of complete graphs $K_{4}, K_{7}, K_{10}, K_{13}$ and $K_{16}$ are shown.
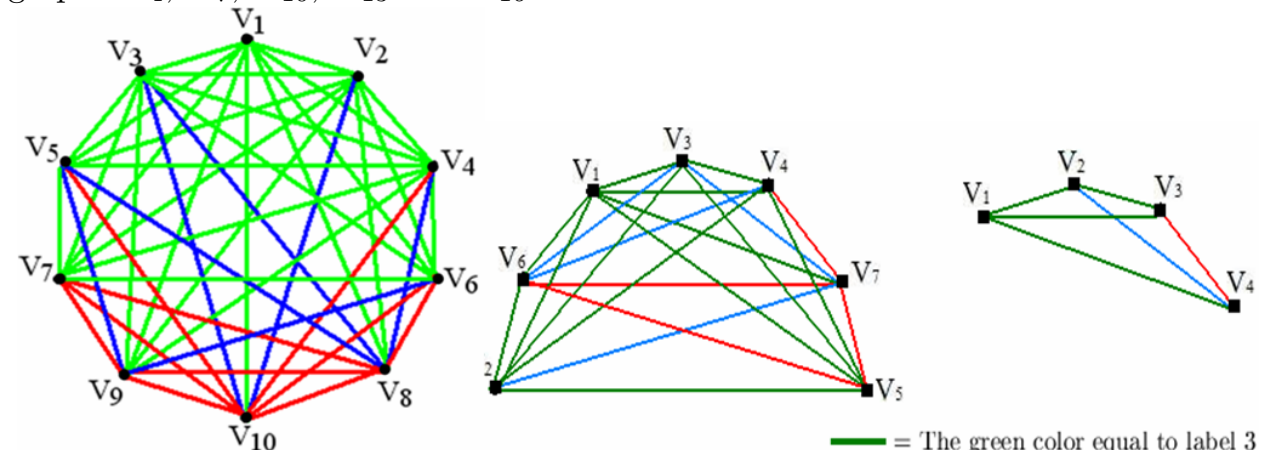

$\mathrm{S}_{1}=27, \mathrm{~S}_{2}=26, \mathrm{~S}_{3}=25, \mathrm{~S}_{4}=24, \mathrm{~S}_{5}=23$,

$\mathrm{S}_{6}=22, \mathrm{~S}_{7}=21, \mathrm{~S}_{8}=20, \mathrm{~S}_{9}=16, \mathrm{~S}_{10}=13$

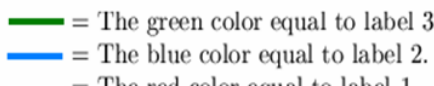

FiguRE 1. The 1-2-3-edge weighting and vertex coloring of complete graphs $K_{4}, K_{7}$ and $K_{10}$.

Thus, the 3-edge weighting $w: E\left(K_{3 q+1}\right) \rightarrow\{1,2,3\}$ and the vertex coloring $s: V\left(K_{3 q+1}\right) \rightarrow\{9 q, 9 q-1, \ldots, 7 q+1,7 q, 7 q-2,7 q-5,7 q-9, \ldots, 3 q+11,3 q+$ $7,3 q+4\}$ is a proper 1-2-3-edge weighting and vertex coloring of $K_{3 q+1} \forall q \in \mathbb{N}$ and this complete the proof of theorem.

By using the proof of Theorem 1.1 (the 3-edge weighting $w$ and the vertex coloring $s)$, one can see that the number of all edge weigh 3,2 and 1 are equal to $\alpha_{3 q+1}=3 q^{2}+1=\left(\left|E\left(K_{3 q+1}\right)_{3}\right|\right), \beta_{3 q+1}=3 q-2=\left(\left|E\left(K_{3 q+1}\right)_{2}\right|\right)$ and $\gamma_{3 q+1}=$ $\frac{3 q^{2}-3 q+2}{2}=\left(\left|E\left(K_{3 q+1}\right)_{1}\right|\right)$. For example, $E\left(K_{3 q+1}\right)_{2}=\left\{v_{2} v_{3 q+1}, v_{4} v_{3 q}, v_{5} v_{3 q-1}\right.$, $v_{5} v_{3 q}, v_{6} v_{3 q-1}, v_{7} v_{3 q-2}, v_{s} v_{3 q-1}, v_{8} v_{3 q-2}, \ldots, v_{2 q-1} v_{2 q+2}, v_{2 q-1} v_{2 q+3}, v_{2 q} v_{2 q+2}$,

$v_{2 q+2} v_{2 q-3}, v_{2 q+2} v_{2 q-2}, v_{2 q+2} v_{2 q-1}, v_{2 q+3} v_{2 q-3}, v_{2 q+3} v_{2 q-2}, v_{2 q+3} v_{2 q-1}, v_{2 q+4} v_{2 q-5}$, $v_{2 q+4} v_{2 q-4}, v_{2 q+4} v_{2 q-3}, \ldots, v_{3 q-2} v_{6}, v_{3 q-2} v_{7}, v_{3 q-2} v_{8}, v_{3 q-1} v_{5}, v_{3 q-1} v_{6}, v_{3 q-1} v_{7}$, $\left.v_{3 q} v_{3} v_{3 q} v_{4}, v_{3 q} v_{5}, v_{3 q+1} v_{2}, v_{3 q+1} v_{3}\right\}$.

\section{Conclusions and Conjectures}

We conclude our paper with the following open questions and conjectures:

Corollary 3.1 (The 1-2-3-conjecture [6, 9]). Every connected graph $G=(V, E)$ non-isomorph to $K_{2}$ (with at least two edges) has an edge labeling $f: E \longrightarrow$ $\{1,2,3\}$ and vertex coloring $S: V \longrightarrow\{n-1, \ldots, 3 n-3\}$.

Corollary 3.2 ( $n$ vertex coloring). There are distinct numbers of $S_{v}$ 's, $v \in$ $V(G)$, of a graph $G$ of order $n$, for a 1-2-3-edge labeling and vertex coloring. 


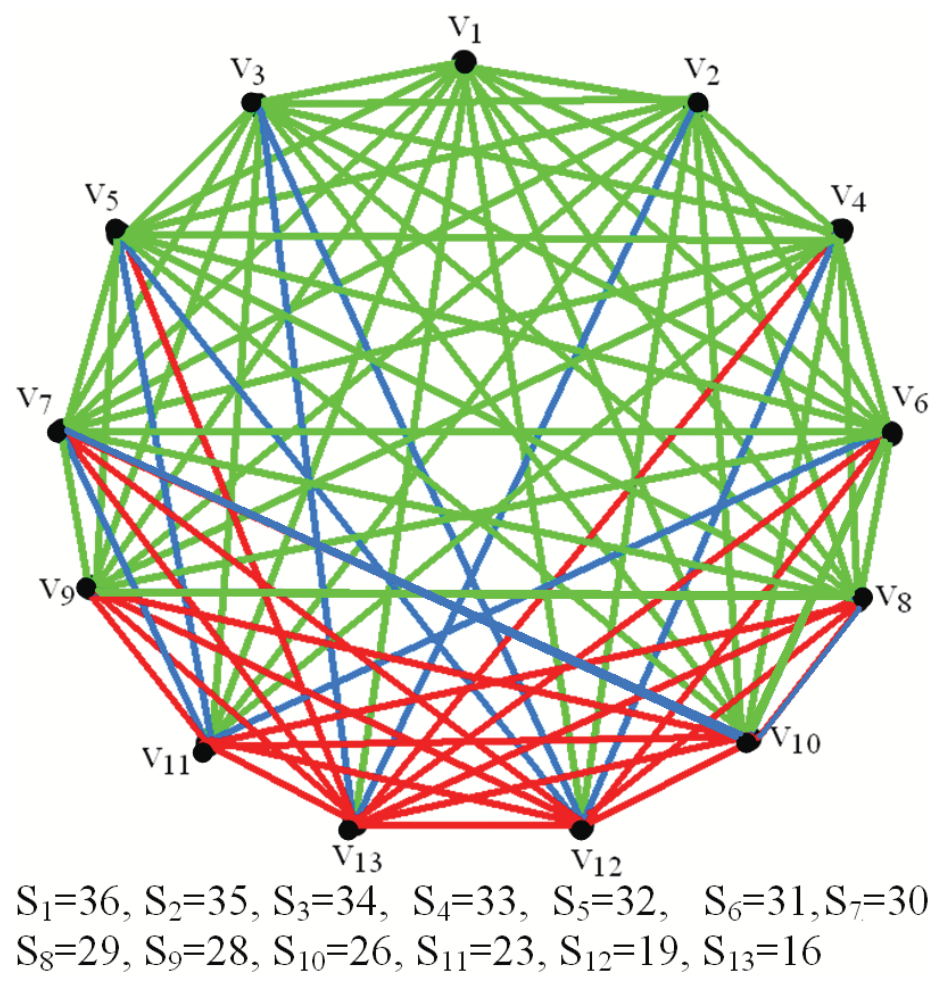

Figure 2. The 1-2-3-edge weighting and vertex coloring of $K_{13}$.

Corollary 3.3 (Proper vertex coloring). For all graph $G$ of order $n$, there are the $\chi(G)$ numbers of $S_{v}$ 's, $v \in V(G)$, with this 1-2-3-edge labeling and vertex Coloring. Where $\chi(G)$ is the number colors of the vertices on the graph $G$.

In this parer, we show that the complete graph $K_{3 q+1}(q \geq 1)$, recognize in three Conjecture 3.1, 3.2 and 3.3.

\section{Acknowledgement}

The authors are thankful to Dr. Mehdi Alaeiyan, Mr Ali Ramin and Mr Seied Hamid Hosseini of Department of Mathematics, Iran University of Science and Technology (IUST) for their precious support and suggestions.

\section{REFERENCES}

1. Addario-Berry, L., Dalal, K., McDiarmid, C., Reed, B.A., and Thomason, A., Vertexcoloring edge-wheitings, Combinatorical. J. 27 (1982), (2007), 1-12.

2. Addario-Berry, L., Dalal, K., and Reed, B.A., Degree constrained sub- graphs,Discrete Applied Mathematics J. 156 (2008), 1168-1174. 


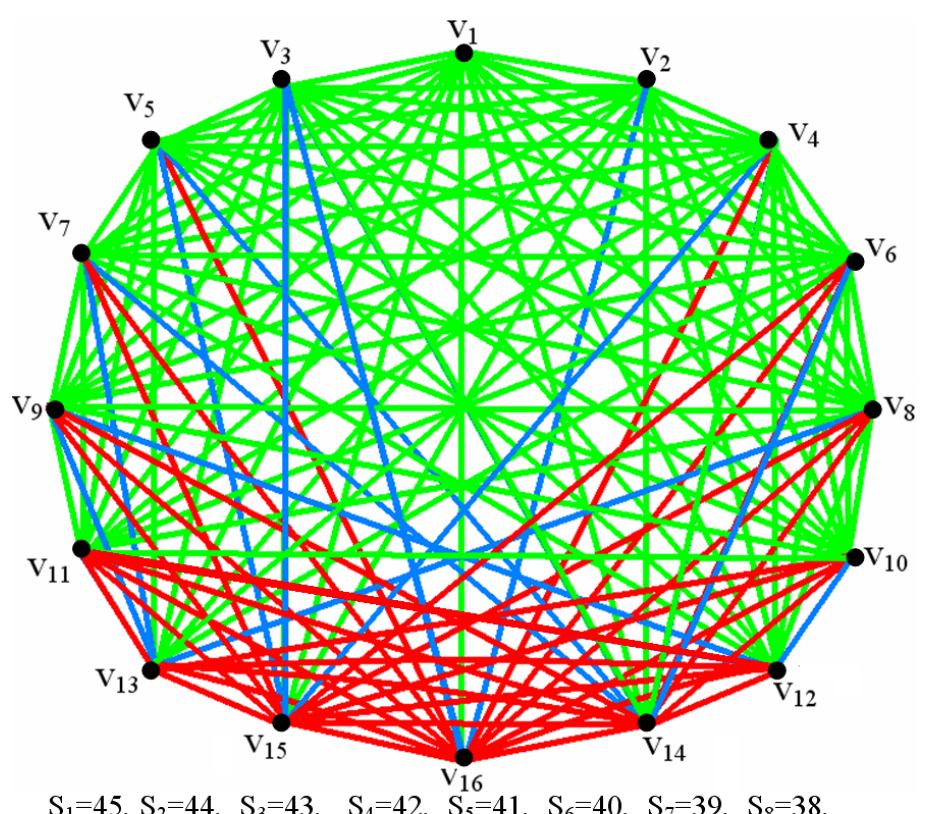

$\mathrm{S}_{9}=37, \mathrm{~S}_{10}=36, \mathrm{~S}_{11}=35, \mathrm{~S}_{12}=33, \mathrm{~S}_{13}=30, \mathrm{~S}_{14}=26, \mathrm{~S}_{15}=22, \mathrm{~S}_{16}=19$

Figure 3. The 1-2-3-edge weighting and vertex coloring of complete graph $K_{16}$.

3. Alaeiyan, M., Farahani, M.R., and Mohammadian, S., The 1-2-3-edge labeling and vertex colors. Submitted for publish, (2013).

4. Farahani, M.R., On The 1-2-3-Edge Weighting and Vertex Coloring of Complete Graph. Int. J. Computational Sciences \& Applications. (2013), Inpress.

5. Farahani, M.R., and Hosseini. S.H., The 1-2-3-edge labeling and vertex coloring of complete graph $K_{n}$. Submitted for publish, (2013).

6. Grytczuka, J., Dalal, K., and Reed, B.A., Thue type problems for graphs, Discrete Mathematics J. 308 (2008), 4419-4429.

7. Kalkowski, M., Karonski, M., and Pfender, F., Vertax-Coloring Edge-Weithings With Integer Weights At Most 6, Rostock. Math. Kolloq. 64 (2009), 39-43.

8. Kalkowski, M., Karonski, M., and Pfender, F., Vertex-coloring edge-weightings: To wards the 1-2-3-conjecture, Combinatorial Theory (Series B) J. 100 (2010), 347-349.

9. Karonskiy, M., Łuczak, T., and Thomason, A., Edge weights and vertex colors, Combinatorial Theory (Series B) J. 91 (2004), 151-157.

10. Wang, T., and Yu, Q., On vertex-coloring 13-edge-weighting, Front. Math. China. 3(4) (2008), 1-7.

\section{Mohammad Reza Farahani}

Department of Applied Mathematics, Iran University of Science and Technology (IUST), Narmak, Tehran 16844, Iran.

e-mail: MR_Farahani@Mathdep.iust.ac.ir 